\title{
An Entropy Approach to Wireless Power Transmission by Magnetic Resonance
}

\author{
Salama Abdelhady ${ }^{1}$ \\ ${ }^{1}$ Energy Engineering College, Aswan University, Aswan, Egypt \\ Correspondence: Salama Abdelhady, Professor of Energy Systems, Energy Engineering College, Aswan \\ University, Aswan, Egypt. E-mail: salama_abdelhady@hotmail.com
}

Received: June 2, 2013 Accepted: June 27, 2013 Online Published: July 31, 2013

doi:10.5539/apr.v5n5p29 URL: http://dx.doi.org/10.5539/apr.v5n5p29

\begin{abstract}
It was previously introduced an entropy approach that identifies the nature of electric current and magnetic flux as a flow of electromagnetic waves that have electric or magnetic potentials. According to such definition, it was possible in a recently published study to find a plausible explanation of the discovered Tesla's "Radiant Energy" as normal transfer of electric current, of wave-nature, through air by the high electric potential of Tesla's tower. In this paper, results of an experimental study and of Faraday's experiment of magnetic induction are used to prove the analogy between the electric energy and magnetic flux as electromagnetic waves of analogous potentials. Accordingly, the MIT discovery of wireless power transmission which was identified as "Evanescent waves" can be plausibly defined as wireless transfer of normal magnetic flux, of wave-nature, through air by the high potential of the discovered magnetic resonant coupling.
\end{abstract}

Keywords: entropy, electromagnetic waves, evanescent waves

\section{Introduction}

Following an entropy approach and reviewing analogies between the thermal, electric and magnetic fluxes, it was possible to prove that the flow of heat, electric charges and magnetic flux have similar natures as electromagnetic waves of analogous potentials (Abdelhady, 2011). In other words, the flow of electric charges should be defined as a flow of electromagnetic waves that have an electric potential and the magnetic flux as a flow of electromagnetic waves that have a magnetic potential similar to the heat radiation which is defined as electromagnetic waves that have a thermal potential. The definition of electric current as electromagnetic waves that have an electric potential was introduced in a recently published paper to find a plausible explanation of the Tesla's discovery of wireless transmission. It was proved that the claimed "Radiant Energy" of Tesla's discovery is the defined flow of electric charges, as waves, through air by the high potential of Tesla's tower (Abdelhady 2013). In the present paper, a review of experimental and analytical proofs that discuss the truth of the definition of magnetic flux as electromagnetic waves, according to an entropy approach, will be presented in the next two sections of this study. A modified analysis of Faraday's experiment of magnetic induction will be considered to prove the analogy between the definitions of the electric current and the magnetic flux as electromagnetic waves of analogous potentials in the fourth section. Then the introduced approach will be applied to analyze the MIT discovery of wireless power transmission by powerful magnetic resonance (Kurs, 2007). Comparisons between the introduced definition of transmitted energy between the two resonant coils as electromagnetic waves that have a magnetic potential and a previously introduced definition of such flux as "evanescent waves" (Cannon, 2009) will be discussed in the fifth section. Conclusions will then be discussed in the last section.

\section{An Entropy Approach to Electric Current and Magnetic Flux}

The flow of electric charges and the magnetic flux were postulated as special solutions of Maxwell's wave equations (Abdelhady, 2011). However, the time in Maxwell's equations is replaced by entropy as an arrow of time which represents a unique property of entropy (Zeh, 1990). So, the modified Maxwell's wave equations read (Abdelhady, 2010a):

$$
\left(\nabla^{2}-\frac{1}{c^{2}} \frac{\partial^{2}}{s^{2}}\right) E=0
$$




$$
\left(\nabla^{2}-\frac{1}{c^{2}} \frac{\partial^{2}}{s^{2}}\right) B=0
$$

Replacement of time by entropy in the previous Maxwell's equations transfers its original space-time frame of reference into an energy frame of reference that depends on entropy as an energy property (Abdelhady, 2012). Accordingly, the flow of electric charge as electromagnetic waves is considered as a special solution of Equations 1 and 2 by introducing the electric potential of the flowing E.M. waves as an initial condition. Such solution reads (Abdelhady, 2012):

$$
\begin{gathered}
E=E_{0}+E_{1} \cos (\omega s) \\
H=H_{1} \cos (\omega s)
\end{gathered}
$$

$E$ and $H$ are the electric and magnetic potentials of the flowing charges; $E_{1}$ and $H_{1}$ represent the electric and magnetic oscillating components of the flowing charge around zero coordinates; $E_{0}$ is the threshold potential of the flowing charges considered as an initial condition of the electric component of the electromagnetic wave, $\omega$ represents the angular velocity of the flowing electromagnetic waves, and $s$ represents the flowing entropy. Graphical representation of such solution is seen in an E-H-s coordinates system in Figure 1 as electromagnetic waves that have a net positive electric potential, $\Delta \mathrm{E}$ (Abdelhady, 2010b). Such approach was introduced in a recently published paper to prove that Tesla's radiant energy is a normal electric flux or electromagnetic waves of electric potential that can be defined according to Equations 3 and 4 (Abdelhady, 2013). Accordingly, the transmitted energy was defined by the following equation:

$$
\dot{\Phi}_{\text {electric }}=E \dot{S}_{\text {electrric }}
$$

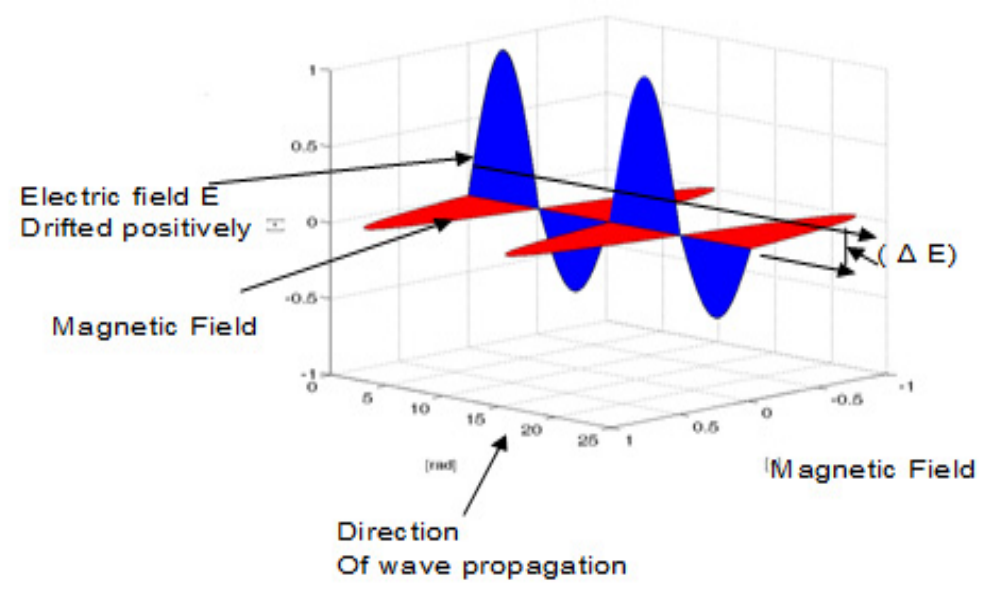

Figure 1. Flow of electric charges as E.M. waves of threshold electric potential

By a similar procedure, the following equations may represent the magnetic flux as special solutions of Equations 1 and 2. In these equations, the magnetic flux is considered as E.M. wave that have an initial magnetic threshold potential " $H_{0}$ " (Abdelhady, 2010):

$$
\begin{gathered}
E=E_{0} \cos (\omega s) \\
H=H_{0}+H_{1} \cos (\omega s)
\end{gathered}
$$

Accordingly, the solution that represents the magnetic flux was represented graphically according to Equations 6 and 7 in the E-H-s coordinates as shown in Figure 2. 


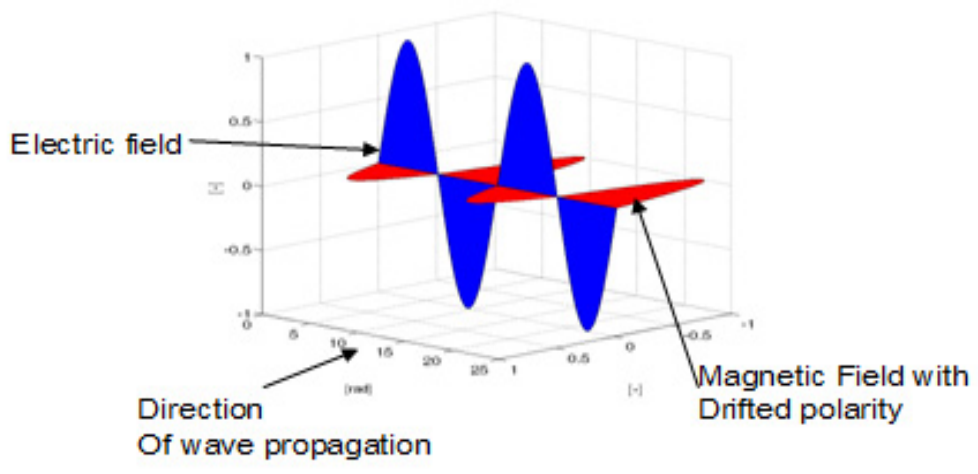

Figure 2. Flow of magnetic flux as E.M. waves of threshold magnetic potential

So, the propagated magnetic energy, $\Phi_{\text {mag }}$, at any position can be represented by the following integral (Abdelhady, 2010):

$$
\Phi_{\text {mag }}=\int H d S_{\text {mag }} .
$$

Such integral is represented by the red-shaded area in the "H-s" coordinates-system as shown in Figure 2. Representation of magnetic energy in an H-s diagram is analogous to representing the heat flow in T-s diagrams as found in thermodynamic literatures (Yunus, 2006). The rate of heat flow is also expressed in thermodynamics as the product of absolute temperature times the rate of entropy flow according to the following equation:

$$
\dot{\mathrm{Q}}_{\text {thermal }}=T \dot{\mathrm{S}}_{\text {thermal }}
$$

By analogy to heat flow; the rate of flow of magnetic energy can be also expressed as follows:

$$
\dot{\Phi}_{\text {magnetic }}=H \dot{\mathrm{S}}_{\text {magnetic }}
$$

\section{An Experimental Approach to the Nature of Magnetic Flux}

Figure 3 shows a magnet immersed in water inside a glass flask. The magnet is attracting iron balls along an inclined smooth glass plane. The temperature of the water surrounding the magnet was measured by a digital thermometer connected to a data logger during the upward motion of the balls along the inclined plane. During the upward attraction of the iron balls, the magnet is performing a mechanical work that results in a decrease of the water temperature as recorded by the digital thermometer. By applying the first law of thermodynamics on the a control mass that includes the insulated basin, i.e. water and magnet system, we get the following equation (Yunus, 2006):

$$
\mathrm{C}_{\mathrm{B}} \Delta t=n m_{b} g d \sin \varphi
$$

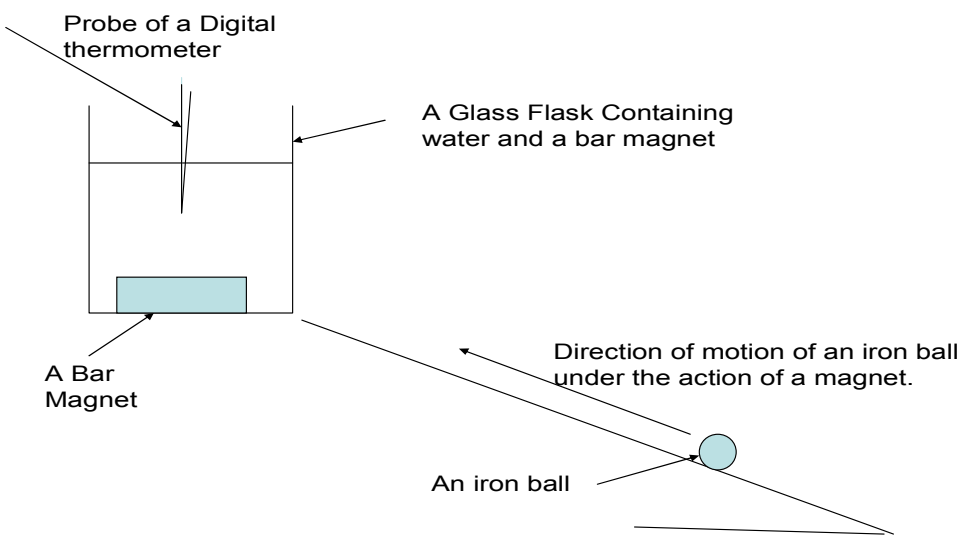

Figure 3. Measurement of Magnet's Work during attraction of iron balls 
Where $C_{B}$ is the basin heat capacity, $n$ is the number of attracted balls along the inclined plane per minute, $m_{b}$ is the mass of the iron balls, $g$ is the acceleration gravity, $d$ is the displacement of the iron ball along the inclined smooth plate $\varphi$ is the angle of inclination of the smooth plate and $\Delta t$ is the increase in water temperature per minute. Reviewing the measurement results, a decrease in water temperature was found during attraction of the iron balls. In other words, the emitted magnetic flux from the magnet that pulls the balls up was substituted by decrease in the temperature of the magnet and the water in the basin. Such results prove the magnetic flux emitted by the magnet can be considered as a categorized form of energy. In other words, the magnetic flux should have the same nature of the absorbed heat from the water. As the heat is originally electromagnetic waves of thermal potential, then the magnetic flux should be defined also electromagnetic waves of a corresponding magnetic potential (Abdelhady, 2010).

\section{Experimental Analogy between Magnetic Flux and Electric Current}

Figure 4 shows Faraday's apparatus that demonstrates interactions between magnetic and electric fluxes (Haaida, 2004). According to this experiment, when the shown switch is closed, a magnetic field is emitted from the coil on the top part of the iron ring, called as a primary coil, and is transmitted to the coil on the bottom part of the ring which is called as a secondary coil. The galvanometer is used to detect any current induced in the secondary coil. It was found that each time the switch is closed; the galvanometer detects a current in one direction in the secondary coil. Each time the switch is opened, the galvanometer detects also a current in the opposite direction.

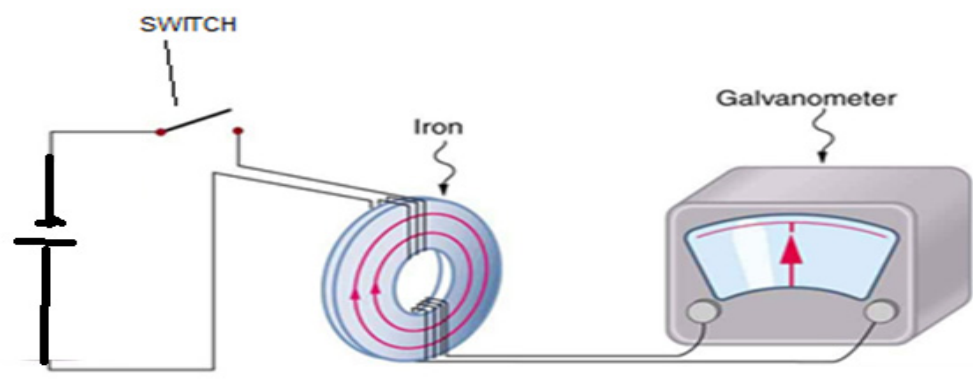

Figure 4. Faraday's apparatus for demonstrating the interactions between electric and magnetic energies

Available literature defines the electric current as a flow of electrons and defines the magnetic flux as the quantity of magnetic field that penetrates an area at right angles (Haaiday, 2004). Defining the electric current as a flow of electrons in the primary coil, it should induce by analogy between the electric current and magnetic flux a flow of analogous particles in the iron ring. Similarly, if the magnetic flux that passes through the secondary coil is defined as the quantity of magnetic field that penetrates an area at right angles, then it should not regenerate a flow of particles in the secondary coil as both have different natures. So, the demonstrated Faraday's experiment shows that both fluxes should have similar natures as both can be interchanged alternatively. However, the previous definitions of electric current and magnetic flux cannot explain such interchangeability. On the other hand, the postulated definitions of electric current and magnetic flux as electromagnetic waves of common nature find a plausible analysis of the demonstrated experiment. As a plausible summary that depends on the postulated definitions, the electric potential in the battery or primary circuit forces electric charges to flow as electromagnetic waves of electric potential in the primary coil. Then such electric potential of the electric current will induce a magnetic potential in the magnetic iron that forces the input electromagnetic waves to outflow as magnetic flux in the iron ring. Then, such magnetic flux flowing through the iron ring induces an electric potential in the secondary coil that forces the input electromagnetic waves to outflow as an electric current in the conducting wires of the secondary coil. Applying the energy conservation principles on the energy transfer processes between the primary coil, the iron ring and the secondary coil, the following equation can be found by expressing the magnetic and electric energies according to Equations 5 and 10:

$$
\left.\left.\left.\mathrm{E} \dot{\mathrm{S}}_{\text {electrical }}\right)_{\text {primary coil }}=H \dot{\mathrm{S}}_{\text {magnetic }}\right)_{\text {iron ring }}=\mathrm{E} \dot{\mathrm{S}}_{\text {electrical }}\right)_{\text {secndary coil }}
$$

The previous equation identifies also the analogy between the electric and magnetic energies. The flow of both energies, electric and magnetic, is expressed as flow of entropy waves by their corresponding potential. Such physical and mathematical analogies as demonstrated by Faraday's results express the truth of the postulated definitions of electric and magnetic fluxes as electromagnetic waves. 


\section{Analysis of Magnetically Coupled Resonant Wireless Power Systems}

In year 2007, a team of MIT published a paper titled "Wireless Power Transfer via Strongly Coupled Magnetic Resonances" (Kurs, 2007). The paper announced a high efficiency mid range wireless power transfer technology that depends on magnetic resonant mutual coupling. Two systems are said to be mutually magnetically or inductively coupled when they are closely configured such that they may exchange energy due to mutual inductance (Cannon, 2009). Figure 5 represents a scheme of the MIT experimental setup (Kurs, 2007).

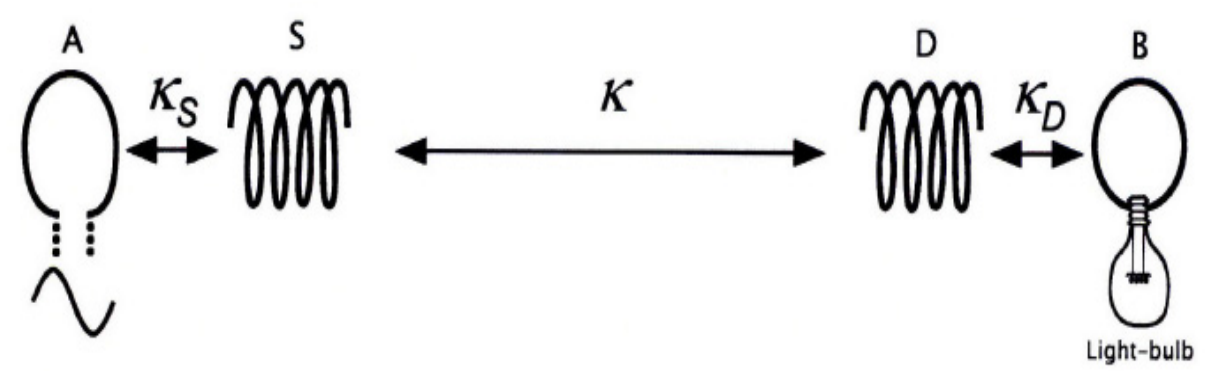

Figure 5. Schematic of the MIT experimental setup

The physical principle behind this system was explained by different authors who defined the waves that allow the energy transfer between the coils of such couplings as "evanescent waves" (Cannon, 2009). They postulated if two resonant circuits are tuned at same frequency, then their near fields are coupled by means of such evanescent waves. They measured the dependence of the received energy and its potential as function of the distance between the source-coil "S" to the receiver coil "D" as seen in Figures 6 (Cannon, 2009) and 7 (Mandip, 2011). According to the seen measurement results in Figures 6 and 7, the evanescent waves are defined as electromagnetic waves that loose part of its transmitted energy and suffer a decay of its potential during its flow through air.

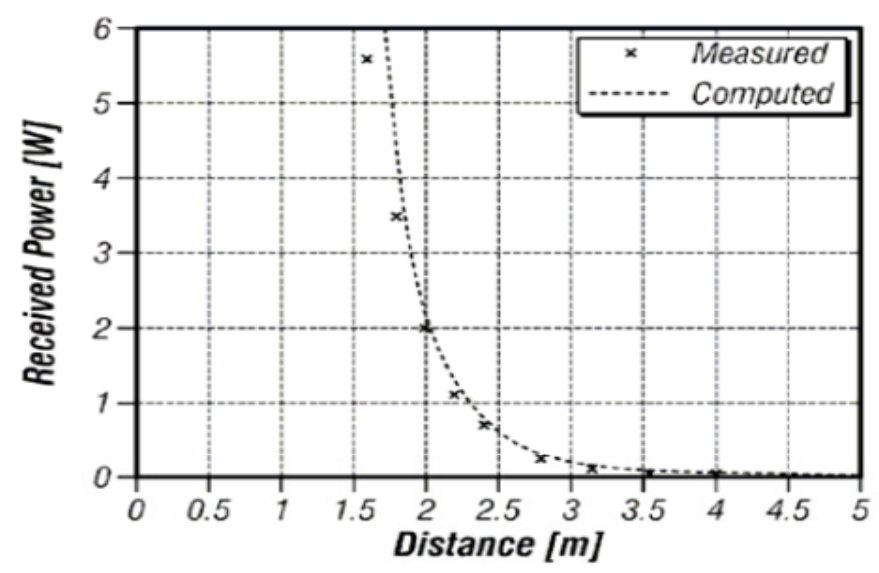

Figure 6. Received Power as a function of the distance of separation of coupling

According to the introduced analysis of Faraday's experiment in the previous section, the emitted energy from the source coil "S" was defined as electromagnetic waves that have a magnetic potential. According to the previous analysis, such electromagnetic waves may be transferred normally though air as a magnetic flux. Then such transmitted flux will be trapped resonantly by the device coil "D" and induce, according to Faraday's law of induction, an electric current through the light-bulb B. 


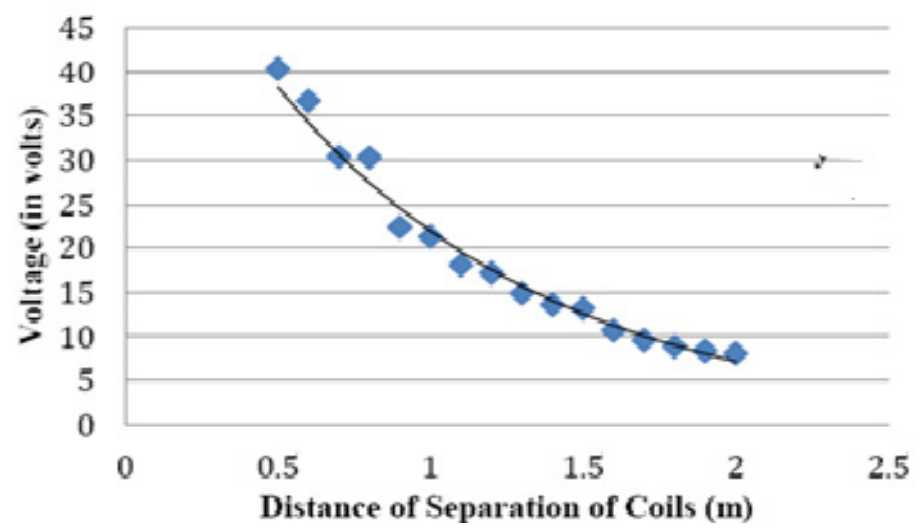

Figure 7. Potential of the received waves

The loss of magnetic potential or the transmitted energy from the source coil to the device coil can be identified as a result of the low permeability or the high reluctance of air that resist the flow of magnetic flux (Haaiday, 2004). Such lost energy is dissipated in air as thermal energy and it can be estimated by applying the energy conservation principles as follows:

$$
\dot{\Phi}_{\text {lost }}=\dot{\Phi}_{\text {source }}-\dot{\Phi}_{\text {device }}
$$

In Equation 13, $\dot{\Phi}_{\text {source }}$ is the rate of energy emission from the source coil, $\dot{\Phi}_{\text {device }}$ is the rate of energy reception in the device coil, and $\dot{\Phi}_{\text {lost }}$ is the rate of loss of the transmitted energy to air as seen in Figure 6 . Such loss of energy led to the measured decay of magnetic potential of the transmitted flux as seen in Figure 7. However, the magnetic potential of the flowing E.M. waves was described in literature by the following equation (Mandip, 2011):

$$
H_{D}=H_{S} e^{-k r}
$$

In Equation 14; $H_{D}$ and $H_{S}$ are the magnetic potentials of the magnetic flux at the device and the source positions respectively; $k\left(\mathrm{~m}^{-1}\right)$ and $r(\mathrm{~m})$ are the wave and position vectors respectively. Such results show that the properties of postulated magnetic flux as electromagnetic waves that have a decaying magnetic potential are consistent with the measured properties of the introduced evanescent waves. So, the evanescent waves are normal magnetic flux that flow as waves through air.

According to the shown results in Figure 7, the unit of measurement of the potential of the emitted magnetic flux is the "Volt." Such measurement unit is consistent with the previously proposed universal unit of magnetic potential (Abdelhady, 2010). So, such measurements sustain also the previously introduced universal system of units that postulated the "Volt" as a unique unit for all potentials, i.e. thermal, electric, magnetic and chemical potentials.

\section{Conclusion and Outlook}

According to an entropy approach and a review of measured and available experimental results, the magnetic flux is defined as a flow of electromagnetic waves that have a magnetic potential similar to the previously defined electric current as a flow of electromagnetic waves that have an electric potential. So, the MIT discovered wireless power transmission through strongly coupled resonances should also be defined as a flow of electromagnetic waves that have a magnetic potential. Such definition identifies the discovered "evanescent waves" as normal magnetic flux or a flow of electromagnetic waves that have a magnetic potential through air due to the high potential of the magnetic resonant coupling.

Clear understanding of magnetic flux as electromagnetic waves may revolutionize a high-efficiency charging system that uses magnetic flux at extremely high or low frequencies to wirelessly transmit high power between metal coils placed several Miles apart. This may represent a successful approach to increase the usage of electric vehicles in general and decrease the world's pollution from classical combustion engines.

\section{References}

Abdelhady, S. (2010a). A Fundamental Equation of Thermodynamics that Embraces Electrical and Magnetic Potentials. Journal of Electromagnetic Analysis \& Applications, 2, 162-166. http://dx.doi.org./10.4236/jemaa.2010.23023/ 
Abdelhady, S. (2010b). An Approach to a Universal System of Units. Journal of Electromagnetic Analysis \& Applications, 2, 549-556, 2010. http://dx.doi.org./10.4236/jemaa.2010.2907/

Abdelhady, S. (2011). Comments on Einstein's Explanation of Electrons, Photons, and the Photo-Electric Effect. Applied Physics Research, 3(2), 230-240. http://dx.doi.org./5539/apr.v3n2p230/

Abdelhady, S. (2012). A Thermodynamic Analysis of Energy Flow in Optical Fiber Communication Systems. Applied Physics Research, 4(3), 22-29. http://dx.doi.org./10.5539/apr.v4np22

Abdelhady, S. (2013). An Entropy Approach to Tesla's discovery of Wireless Power Transmission. Journal of Electromagnetic Analysis \& Applications, 5, 157-161. http://dx.doi.org./10.4236/jemaa.2013.54025/

Cannon, B., Hoburg, J., Stancil, D., \& Goldstein, S. (2009). Magnetic resonant coupling as a potential means for wireless power transfer to multiple small receivers. Power Electronics, IEEE Transactions on, 24(7), 1819-1825. http://dx.doi.org/10.1109/TPEL.2009.2017195

Haaiday, D., Resnick, R., \& Walker, J. (2004). Fundamentals of Physics (7th ed.). New York, NY: John Wiley \& Sons, 2004.

Kurs, A. (2007). Power Transfer Through Strongly Coupled Resonances (M.Sc. thesis, Massachusetts Institute of Technology, 2007).

Mandip, J. S., \& Hambleton, J. (2011). Wireless Power Transmission Using Magnetic Resonance. Professor Derin Sherman, Cornell College PHY312; 2011. http://www.cornellcollege.edu/physics/files/mandip-sibakoti.pdf

Simpson, F., \& Bahr, K. (2005). Practical Magnetotellurics. Cambridge University Press.

Yunus, A. C., \& Michael, A. B. (2006). Thermodynamics; An Engineering Approach. Boston: McGraw-Hill Scientific Engineering.

Zeh, H. D. (1990). The Physical Basis of the Direction of Time. Science, 249(4965), 192-193.

\section{Copyrights}

Copyright for this article is retained by the author(s), with first publication rights granted to the journal.

This is an open-access article distributed under the terms and conditions of the Creative Commons Attribution license (http://creativecommons.org/licenses/by/3.0/). 